\title{
Matrix compatibility of typical sol-gel solid-phase microextraction coatings in undiluted plasma and whole blood for the analysis of phthalic acid esters
}

\author{
Xiao-Wei Zhang ${ }^{1} \cdot$ Yao-Juan Chu ${ }^{1} \cdot$ Yu-Hao Li ${ }^{1} \cdot$ Xiu-Juan $\mathrm{Li}^{1}$
}

Received: 8 October 2021 / Revised: 8 December 2021 / Accepted: 10 January 2022 / Published online: 16 February 2022

(c) Springer-Verlag GmbH Germany, part of Springer Nature 2022

\begin{abstract}
Sol-gel materials have been widely used for solid-phase microextraction (SPME) coatings due to their outstanding performance; in contrast, sol-gel SPME coatings have seldom been used for in vivo sampling. The main reason is that their matrix compatibility is unclear. In order to promote the application of this type of coating and accelerate the development of in vivo SPME, in this study, the matrix compatibility of several typical sol-gel coatings was assessed in plasma and whole blood using phthalic acid esters as analytes. The service life of five kinds of sol-gel coatings was among 20-35 times in undiluted plasma, while it was 27 times for a homemade commercial polydimethylsiloxane coating, which indicates good matrix compatibility of sol-gel coatings in untreated plasma. The sol-gel hydroxy-terminated silicone oil/methacrylic acid fiber achieved the highest extraction ability among all of the fibers, and it was tested in pig whole blood. It could be continuously used for at least 22 times, demonstrating good potential for in vivo sampling. Subsequently, a direct-immersion SPME/gas chromatography-flame ionization detection method was established for the determination of 5 phthalic acid esters in blood. Compared with other methods reported in the literature, this method is rapid, simple, sensitive, and accurate, and does not need expensive instruments or tedious procedures. A simulation system of animal blood circulation was constructed to verify the practicability of sol-gel SPME coatings in animal vein sampling. The result illustrated the feasibility of that coating for in vivo blood sampling, but a more accurate quantification calibration approach needs to be explored.
\end{abstract}

Keywords Solid-phase microextraction $\cdot$ Sol-gel coatings $\cdot$ Matrix compatibility $\cdot$ Blood $\cdot$ In vivo solid-phase microextraction $\cdot$ Phthalic acid esters

\section{Introduction}

In vivo sampling is currently an important research topic in the field of analytical chemistry [1,2]. It is non-invasive or less invasive toward a subject, and allows for real-time monitoring of samples including living systems in natural environments, and consequently can reflect more accurately the organismal physiological and biochemical processes under

Xiao-Wei Zhang and Yao-Juan Chu contributed equally to this paper.

Xiu-Juan Li

lixiujuan@mail.hzau.edu.cn; lixj78@126.com

1 Key Laboratory of Environment Correlative Dietology (Ministry of Education), College of Food Science \&Technology, Huazhong Agricultural University, Wuhan 430070, China the nature condition [1]. It is especially necessary for unstable compounds and unstable status [3]. Moreover, in vivo sampling allows multiple samplings of the same system over time, which reduces the interorganism variation and saves cost [4]. In recent years, solid-phase microextraction (SPME) is becoming a promising in vivo sampling technique owing to its ultrasimplified workflow, miniaturized extraction assembly, nonlethal sampling characteristic, minor disturbance to living organisms, and many other advantages [1-4]. It has been applied for in vivo sampling of animals, insects, plants, microorganism, and cells [5]. Although significant progress has been made, the application of in vivo SPME still faces challenges including the development of matrix-compatible coatings and the establishment of quantitative calibration methods $[6,7]$.

Blood is the most commonly used biological fluid in clinical diagnosis. During the analysis, complex matrix compositions, enzymatic reactions, possible instability of some 
analytes, $\mathrm{pH}$ variations, storage conditions, and the addition of anticoagulants may affect the integrity of samples [6]. Therefore, whole blood tests are more clinically persuasive than plasma and serum tests. In vivo blood SPME sampling eliminates the blood draw step, extracts a little analytes in a short time, and reduces the risk of analyte degradation or transformation during sample pretreatment [8]. Accordingly, in contrast to in vitro whole blood analysis, the result of in vivo blood sampling is more accurate and more convincing. This technique is especially precious for small animals and unstable compounds. However, during direct sampling of whole blood, coating fouling often occurs due to the presence of proteins, blood cells, and other substances [9]. Therefore, matrix-compatible SPME coatings are required to provide satisfactory recoveries and good repeatability in this complex matrix. This is the first problem to be solved for in vivo blood SPME sampling.

Up to now, some biocompatible materials, such as polydimethylsiloxane (PDMS) [10, 11], polypyrrole [8, 12-15], poly(ethylene glycol) [16, 17], polyacrylonitrile (PAN) [9, 18-22], fluoropolymers [23], chitosan [24, 25], collagen [26], and sol-gel coatings [27-31], have been reported as extraction phases for direct-immersion SPME (DI-SPME) for the determination of different analytes in blood, serum, and plasma. However, only a few coating chemistries, such as polypyrrole [12-15], poly (ethylene glycol) [16, 17], PAN [19], and collagen [26], are used for in vivo blood sampling; even though, various drawbacks have hindered their further implementation [6,32].

Among those extraction phases, PAN-based coatings are the most widely used materials for whole blood analysis due to their anti-fouling performance and amenability to various coating geometries/supports/tunable sorbent chemistry, but they still have some limitations. PAN-based SPME coatings are mainly used for solvent-assisted desorption, and are incompatible with thermal desorption conditions during gas chromatography (GC) analysis due to the thermal degradation of PAN [23]. Polypyrrole and poly(ethylene glycol)based coatings also have the same limitation. PDMS-based coatings are capable of withstanding typical thermal desorption temperatures, but they usually present bleeding peaks, and may swell when in contact with certain solvents $[6,23]$. Furthermore, PDMS-based coatings are mainly used for extraction of nonpolar substances.

Sol-gel technology is widely used for preparing SPME coatings [33, 34]. Numerous significant advantages of sol-gel coatings are reported, namely, easy preparation, high thermal and chemical stability, long lifetime, high surface area, various monomers, possibility to design the material structure, and property by varying the proportion of the sol-gel solution [34]. Furthermore, sol-gel functional hybrid materials are nontoxic and noninjurious to living system and have been widely used for biomedical applications such as scaffolds and matrices for tissue repair and regeneration, nanocarriers for delivering biomolecules, and diagnostic purposes [35]. All of these advantages show the great potential of sol-gel coatings in in vivo SPME application.

So far, the sol-gel coatings have been used for serum and plasma samples for various purposes. Zilfidou et al. developed a fabric phase adsorption extraction membrane coated with a sol-gel adsorbent, and it had a reusability of at least 30 times for extraction of 5 antidepressant drug residues from human serum [27]. Sol-gel coatings also have excellent reusability in more complex plasma samples. Mu et al. developed a sol-gel aptamer SPME fiber to capture adenosine from undiluted human plasma, and the fiber could be used over 20 cycles without significant loss of efficiency [28]. El-Beqqali et al. reported a molecularly imprinted sol-gel tablet for micro-solid-phase extraction of methadone in human plasma, and each tablet could be used for twenty times [29]. When the plasma is diluted, longer lifetime could be obtained. Moein et al. prepared a needle trap utilizing a molecularly imprinted sol-gel xerogel for on-line microextraction of bilirubin from five times diluted plasma, and the needle could be used for approximately one hundred extractions [30]. Recently, a novel SPME fiber based on a capillary glass tube coated with magnetic $\mathrm{Fe}_{3} \mathrm{O}_{4} / \mathrm{Cu}_{3}(\mathrm{BTC})_{2}$ metal organic framework nanocomposites was prepared using sol-gel matrix as dispersant and adhesive [31]. The fiber was successfully applied for determination of non-steroidal anti-inflammatory drugs in real samples including human urine, serum, plasma, and tablet formulation. These studies illustrate the matrix compatibility of sol-gel SPME coatings in serum and plasma; however, within our knowledge, there is few literature reported on the use of sol-gel SPME fibers for whole blood sampling.

In order to investigate the matrix compatibility of sol-gel SPME coatings, and promote the development of in vivo SPME sampling, in this study, several sol-gel SPME coatings were carefully investigated in plasma and whole blood with phthalic acid esters (PAEs) as model analytes. PAEs are a class of compounds that have been extensively used as plasticizers in different applications. They enter human body and animals through a variety of ways, causing great threats and harm to their lives and health [36]. The implementation of pre- and post-desorption rinsing procedures was significant to extend the coating lifetime. After that, a direct-immersion solidphase microextraction-gas chromatography (DI-SPME/ GC) method was established for analyzing PAEs in whole blood. Finally, a simulation system of animal blood circulation was constructed to explore the applicability of sol-gel coatings for sampling in animal veins, which is the upfront work of in vivo sampling of living animals. 


\section{Materials and methods}

\section{Chemicals, standard solutions, and samples}

Analytical standards (purity greater than 98\%) of dimethyl phthalate (DMP), diethyl phthalate (DEP), dibutyl phthalate (DBP), benzyl butyl phthalate (BBP), and di (2-ethylhexyl) phthalate (DEHP) were obtained from Shanghai Aladdin Bio-Chem Technology Co., Ltd. (Shanghai, China), and detailed information of these analytes is given in Table S1 (Supplementary information). Trifluoroacetic acid (TFA, 99\%), n-vinylpyrrolidone (NVP), divinylbenzene (DVB), butyl methacrylate (BMA), and methacrylic acid (MAA) were purchased from the same company. Methanol, acetonitrile, toluene, and acetone were analytically pure and purchased from Sinopharm Chemical Reagent Co., Ltd. (Shanghai, China). Hydroxy-terminated silicone oil (OH-TSO), poly(methylhydrosiloxane) (PMHS), tetraethoxysilane (TEOS), and 3-(trimethoxysilyl)propyl methacrylate (KH-570) were obtained from Hubei Wuhan University Organic Silicone New Material Co., Ltd. (Wuhan, China). Sylgard 184® (PDMS prepolymer and curing agent) was purchased from Dow Corning (Midland, MI, USA). Hydrophilic-lipophilic balanced (HLB) particles were synthesized according to Vasiljevic et al. [37]. Ultrapure water was obtained from a Millipore-Q ultrapure water system (Massachusetts, USA). Stainless steel wire $(80 \mu \mathrm{m}$ o.d.) was purchased from Jinsanshun Stainless Steel Wire Co., Ltd. (Dongguan, China).

A mixture of stock solution containing DMP (5 mg mL $\left.{ }^{-1}\right)$, DEP $\left(2 \mathrm{mg} \mathrm{mL}^{-1}\right)$, DBP $\left(1 \mathrm{mg} \mathrm{mL}^{-1}\right)$, BBP $\left(1.5 \mathrm{mg} \mathrm{mL}^{-1}\right)$, and DEHP $\left(10 \mathrm{mg} \mathrm{mL}^{-1}\right)$ was prepared by dissolving the above-mentioned analytical standards in methanol. Mixed standard working solutions were prepared by serial dilution of the stock solution with methanol. The stock solution was stored at $-18^{\circ} \mathrm{C}$, while all of the working solutions were stored at $4{ }^{\circ} \mathrm{C}$.

Fresh whole pig blood (EDTA- $\mathrm{K}_{2}$ as anticoagulant) was obtained from a slaughter house in Wuhan. All experimental protocols were approved by the Animal Ethics Committee of Huazhong Agricultural University. Plasma was isolated from fresh whole blood by centrifugation (3000 $\mathrm{r} \min ^{-1}$ for $10 \mathrm{~min}$ at $4{ }^{\circ} \mathrm{C}$ ). The whole blood was maintained at $4{ }^{\circ} \mathrm{C}$ for a maximum of 1 week and plasma was kept frozen at $-80^{\circ} \mathrm{C}$ until use. Before using, the sample was brought to room temperature.

\section{Apparatus and chromatographic conditions}

The experiments were performed on an SP-6890A capillary GC system (Shandong Lunan Ruihong Chemical
Engineering Instrument Co., Ltd., Tengzhou, China) equipped with a capillary splitless injector and a flame ionization detector (FID). A personal computer equipped with a chromatopac model N2000 (Hangzhou Mingtong Technology Co., Ltd., Hangzhou, China) was used to process chromatographic data. Compounds were separated on an SE-54 capillary column $(30 \mathrm{~m} \times 0.32 \mathrm{~mm} \times 0.33 \mu \mathrm{m}$, Lanzhou ATECH Technologies Co., Ltd., Lanzhou, China). The oven temperature programming was as follows: initial temperature, $100{ }^{\circ} \mathrm{C}$; increased to $280{ }^{\circ} \mathrm{C}$ at $15{ }^{\circ} \mathrm{C} \mathrm{min}{ }^{-1}$, held for $6 \mathrm{~min}$. The temperatures of the injector and the detector were set at $280{ }^{\circ} \mathrm{C}$. Nitrogen $(99.999 \%)$ was used as carrier gas and kept at a linear velocity of $12-15 \mathrm{~cm} \mathrm{~s}^{-1}$ for all of the analyses.

Identification of the extracted analytes in blood was performed in an Agilent 6890 N GC coupled to an Agilent 5979B mass selective detector with a HP-5 capillary column ( $30 \mathrm{~m} \times 0.25 \mathrm{~mm} \times 0.25 \mu \mathrm{m}$, Agilent Technologies). The carrier gas was helium at a flow rate of $1.2 \mathrm{~mL} \mathrm{~min}^{-1}$. A split/ splitless injector was used in the splitless mode, and the injector temperature was $280{ }^{\circ} \mathrm{C}$. The mass detector operated in the electron impact mode at $70 \mathrm{eV}$ in a range from 45 to $550 \mathrm{amu}$, and the ion source temperature was set at $230{ }^{\circ} \mathrm{C}$. The oven temperature programming was the same as GC-FID. Peaks were identified by comparing their mass spectra to the NIST 05 library (matching quality higher than 90\%), and further identified using authentic standards.

A magnetic stirrer DF-101S (Zhengzhou Greatwall Scientific Industrial and Trading Co., Ltd., Zhengzhou, China) was employed for stirring the sample during extraction. 24G disposable venous indwelling needles were purchased from Shandong Haidike Medical Products Co., Ltd. (Heze, China). Sterile syringes were purchased from Wuhan Hengkang Medical Instrument Co., Ltd. (Wuhan, China). BT100 peristaltic pump (including PTFE tube, $0.8 \mathrm{~mm}$ i.d., $3.8 \mathrm{~mm}$ o.d., and $40 \mathrm{~cm}$ of length) was attained from Shanghai Qingpu Huxi Instrument Factory (Shanghai, China).

\section{Preparation of SPME fibers}

See Supplementary information.

\section{DI-SPME procedure}

Eight grams of whole blood (or plasma) and a magnetic stir bar were added into a $10-\mathrm{mL}$ glass vial. The vial was then sealed and put in the magnetic stirrer for 15 min incubation at $37{ }^{\circ} \mathrm{C}$ with a stirring rate of $1000 \mathrm{r} \mathrm{min}^{-1}$. DI-SPME was conducted for $40 \mathrm{~min}$ at the same condition. After extraction, the fiber was rinsed for $10 \mathrm{~s}$ and gently dried with Kimwipe tissue. Desorption was carried out for $15 \mathrm{~min}$ at $280{ }^{\circ} \mathrm{C}$, which was followed by a wash step and subsequent cleaning with Kimwipe tissue being soaked in acetone. The rinsing 
and washing conditions are described in Table S2 (Supplementary information). For sampling of the diluted sample, a portion of plasma was thoroughly mixed with four portions of ultrapure water, and $8 \mathrm{~g}$ of this mixture was submitted to DI-SPME.

\section{Simulation of in vivo SPME sampling}

In order to verify the practicability of sol-gel SPME coatings for animal vein sampling, a simulation system of animal blood circulation was constructed (Fig. 1).

A peristaltic pump (1) set at a flow rate of $10 \mathrm{~mL} \mathrm{~min}{ }^{-1}$ functioned as an artificial heart [38], and a PTFE tube acted as an artificial vein. Fresh anticoagulated pig blood $(8 \mathrm{~g})$ was placed in a matrix reservoir (2) and the temperature was controlled at $37^{\circ} \mathrm{C}$ in a water bath. A venous indwelling needle (3) was implanted into the simulated vein. During sampling, a 1-mL disposable sterile syringe (4) was inserted into the heparin cap to manually push and pull the blood at a sampling rate of $1.4 \mathrm{~mL} \mathrm{~min}{ }^{-1}$, and a self-made SPME device (5) was also inserted for extraction of $5 \mathrm{~min}$. Rinsing and desorption steps were performed using the same conditions as described for the optimized procedure.

\section{Quantitative calibration for DI-SPME/GC method}

Calibration samples were prepared by spiking an appropriate volume of different standard solutions into whole blood. Spiked blood samples were incubated overnight in a refrigerator at $4{ }^{\circ} \mathrm{C}$ to permit protein binding and then extracted in DI-SPME mode under the optimized conditions. A plot of the responses versus the concentrations of analytes was then developed, and the unknown concentration initially present in the sample was calculated by extrapolation, which was the $x$-intercept in the plot.
Recoveries were obtained by standard addition method. Three levels of known quantities of PAEs were spiked to pig whole blood. Each of the samples was analyzed by the developed DI-SPME/GC method, and its concentration was then calculated according to the above linear standard curves. The recovery value was obtained by the ratio of the calculated concentration to the spiked one.

\section{Quantitative calibration for in vivo SPME/GC method}

The sampling rate calibration method was used for quantitative calibration [1]. The concentration of analytes in the sample matrix $\left(C_{0}\right)$ can be obtained according to Eq. (1).

$C_{0}=n / R_{s} t$

where $R_{S}$ is the sampling rate for an analyte, and $n$ is the amount extracted by the SPME fiber at time $t$. Before the quantification of $C_{0}$, the sampling rate for a targeted analyte was pre-determined using the same equation where $C_{0}$ was obtained by DI-SPME/GC method, and $n$ was obtained by liquid injection of a series of standard solutions with different concentrations of analytes. And then, the calculated $R_{S}$ was used for quantification of other samples.

\section{Results and discussion}

More and more SPME coatings are prepared via sol-gel technology because of their outstanding properties [33]. The OH-TSO coating represents the basic chemical structure of sol-gel coatings, and its main chemical composition is polydimethylsiloxane, just like the commercial PDMS coating. It has good extraction ability to nonpolar analytes. DVB is often added to enhance the extraction capacity to
Fig. 1 Schematic of the flow system for animal blood circulation (a) and in vivo sampling (b)

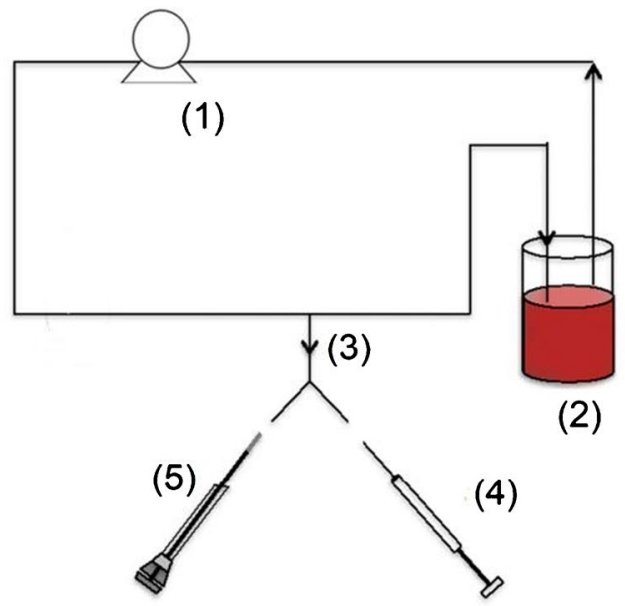

(a)

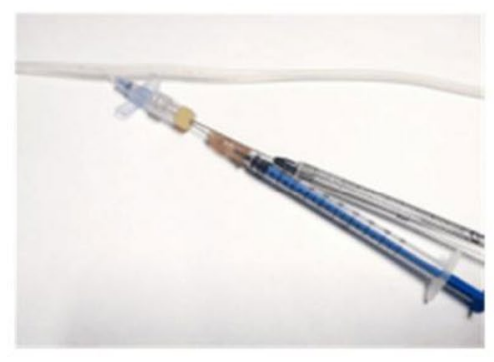

(b) 
unsaturated compounds through $\pi-\pi$ interaction. MAA is a paramount functional monomer for sol-gel coatings and molecularly imprinted coatings to improve the extraction efficiency to polar compounds through hydrogen bonding and dipole-dipole interactions. BMA is less polar than MAA, but its film-forming ability is better than that of MAA, and then it is often used as a substitute for MAA. HLB is a good material to cover a lot of analytes regardless of polarity [39]. Consequently, these five kinds of sol-gel coatings were selected to investigate their matrix compatibility in blood. The preparation and characteristics of these fibers were well described previously [40-42].

PAEs are one of the most frequently detected persistent organic pollutants in the environment. Several of them have been shown to cause negative health effects to animals and humans and their use is restricted in most countries. PAEs are a class of compounds with similar structure, but exhibit a large variety of physicochemical properties due to different carbon chains. Table S1 (Supplementary information) shows the physicochemical properties of five analytes tested in this paper. The logarithm of the octanol-water partition coefficients $\left(\log \mathrm{K}_{\mathrm{OW}}\right)$ shows a wide range, from 1.60 for the rather hydrophilic DMP to 7.60 for the very hydrophobic DEHP, which covers the $\log \mathrm{K}_{\mathrm{OW}}$ of most of analytes for GC instrument [39]. In addition, the solubility also varies greatly, from 4000 to $0.27 \mathrm{mg} \mathrm{L}^{-1}$. In the structure, PAEs compounds contain unsaturated benzene ring, polar ester group, and nonpolar alkyl group, which are the most typical groups for organic compounds. As a result, these PAEs are selected as model analytes to investigate the performance of the sol-gel coatings.

\section{Exploration of pre-desorption rinsing and post-desorption washing conditions of sol-gel OH-TSO coating in blood and plasma}

Previous research has shown that a cleaning procedure including a rapid rinsing of the fiber after extraction and a post-desorption washing after desorption plays an important role in protecting SPME coatings from fouling as a result of direct exposure to complex matrices [43, 44]. Following these recommendations, the rinsing and washing conditions, summarized in Table S2 (Supplementary information), were investigated based on the sol-gel OH-TSO coating.

As shown in Fig. S1 (Supplementary information), methods 1-3 did not give good results. The amounts extracted increased at first, and then decreased dramatically. At the beginning, the unpolymerized components in the coating might be eluted and some absorption sites were released, and thereby the extraction capacity of the coating increased. However, as the number of extraction times increased, blood cells, proteins, and other substances in whole blood adhered to the surface of the coating, and were further solidified on the surface during the high-temperature desorption, which consequently decreased the extraction capacity. Fig. S1 (Supplementary information) declared that the cleaning procedures of these methods could not effectively remove the fouling, but method 3 showed some positive effect on the cleaning because the peak areas declined more slowly than those using the other two methods. Acetone probably cleared away some of the residues since it demonstrated excellent capacity to remove the fouling characterized by high content of sugar, fat, and proteins [43, 44]. Unfortunately, the whole blood sample in the laboratory was used up, and it was difficult to obtain due to the occurrence of African swine fever and COVID-19. The subsequent cleaning procedure had to be investigated in plasma preserved in an ultracold storage freezer.

As listed in method 4 (Table S2, Supplementary information), the post-desorption washing time was extended to $3 \mathrm{~min}$, and simultaneously, the plasma was diluted with 4 times of ultrapure water to reduce matrix interference and guarantee good repeatability during the test. Most of the compounds showed acceptable repeatability during the first 10 extractions. Except DEHP, the RSD of the peak areas of other analytes was less than 7\%. The relative amounts extracted by the fiber of the other nine extractions were between 83.92 and $115.86 \%$ compared to the first one. However, the extraction capacity declined significantly after 20 consecutive extractions (Fig. S1d). After each postdesorption washing, the coating was gently wiped using acetone-soaked Kimwipe tissue. Fig. S2 (Supplementary information) showed that most of the debris attached to the coating surface was cleaned. In method 5, a mixture of water and $0.1 \mathrm{~mol} \mathrm{~L}^{-1}$ hydrochloric acid solution was used for washing. Under this condition, the fiber showed satisfactory repeatability during 20 extractions with RSD from 4.7 to $7.8 \%$ except DEHP.

\section{Lifetime and extraction ability of sol-gel coatings in undiluted plasma}

In the above experiments, the OH-TSO coating showed acceptable repeatability in diluted plasma with method 5 . Considering the possible potency of method 5 in undiluted plasma, Fig. S3 (Supplementary information) evaluated 52 consecutive extractions in undiluted matrix. During the first 35 extractions, the amounts extracted of 5 PAEs did not change a lot, and the RSD $(n=35)$ for each analyte varied within 4.4-19.6\%. After 52 consecutive extractions, the fiber was still good for extraction of DEP and DBP, with a RSD of $9.9 \%$ and $11.1 \%$, respectively. The lifetime of $\mathrm{OH}-$ TSO coating in undiluted plasma is longer than that of the aptamer sol-gel SPME coating [28] and the molecularly imprinted sol-gel tablet coating [29], but shorter than that of the C18-PAN thin-film coating [9]. The latter could be 
Table 1 The lifetimes of 6 coatings in undiluted plasma and the RSDs of their consecutive extractions of 5 PAEs

\begin{tabular}{llrrrrr}
\hline Coating & $\begin{array}{l}\text { Extraction } \\
\text { numbers }\end{array}$ & \multicolumn{2}{l}{ RSD $(\%)$} & & & \\
\cline { 3 - 7 } & & DMP & DEP & DBP & BBP & DEHP \\
\hline OH-TSO & 35 & 19.61 & 11.44 & 4.74 & 4.40 & 16.45 \\
OH-TSO/MAA & 28 & 7.01 & 9.03 & 7.70 & 14.80 & 14.30 \\
OH-TSO/MAA/HLB & 25 & 23.10 & 13.69 & 6.73 & 10.49 & 20.66 \\
OH-TSO/DVB & 21 & 13.08 & 8.93 & 4.56 & 10.04 & 15.83 \\
OH-TSO/DVB/BMA & 20 & 19.47 & 13.32 & 19.74 & 13.21 & 26.34 \\
Self-made PDMS & 27 & 26.57 & 32.33 & 12.38 & 21.73 & 26.57 \\
\hline
\end{tabular}

used at least 70 times for human plasma analysis. Due to the incompatibility with thermal desorption, the PAN-based coatings are desorbed by solvents. During the solvent desorption process, substances attached to the surface of the coating can be further removed, thereby extending the lifetime of the coating. In this study, PAEs were desorbed at high temperature, which caused the impurities to bind more firmly to the coating surface, thereby affecting its reusability.

In order to investigate the matrix compatibility of other sol-gel SPME coatings in undiluted plasma, 3 commonly used sol-gel fibers were prepared, namely OH-TSO/MAA, $\mathrm{OH}-\mathrm{TSO} / \mathrm{DVB}$, and OH-TSO/DVB/BMA fibers. Given the good anti-matrix interference performance of PDMS materials, PDMS probes were also coated and used as a comparison. Recently, SPME extraction phase made of HLB particles immobilized with PAN was used because of its excellent extraction ability to a wide range of analytes [37, 39], and therefore, OH-TSO/MAA/HLB fiber was prepared and applied to plasma matrix. The results for those coatings are summarized in Table 1.

The lifetime is defined according to the RSD of a group of consecutive extractions (below or around 20\%) and the extraction capacity (the extraction capacity does not decrease dramatically). As is shown, the OH-TSO coating had the longest lifetime in undiluted plasma. The service life of $\mathrm{OH}-$ TSO/MAA coating was 28 times in plasma, and the surface of the coating was smooth after being wiped with acetonesoaked Kimwipe tissue. Besides, the repeatability was better than that of the OH-TSO coating. The surface of OH-TSO/ MAA/HLB fiber was relatively rough because HLB particles were blended in the coating. After being wiped by the tissue, the coating became thinner, the extraction capacity decreased, and the service life decreased. The sol-gel OHTSO/DVB fiber was not easy to be coated, and the surface was not as smooth as those of the OH-TSO/MAA and OHTSO coatings, which affected its repeatability and lifetime.
Fig. 2 Comparison of the extraction capability of different coatings to 5 PAEs in plasma. Spiked concentration: $125 \mu \mathrm{g} \mathrm{kg}^{-1}$ for each PAEs. Extraction and desorption conditions: extraction time, $30 \mathrm{~min}$ extraction temperature, $37^{\circ} \mathrm{C}$; desorption temperature, $280{ }^{\circ} \mathrm{C}$; desorption time, $10 \mathrm{~min}$; cleaning condition, method 5. Different letters indicate that there is a significant difference in the groups $(p<0.05)$, and the same letter indicates that there is no significant difference $(p>0.05)$

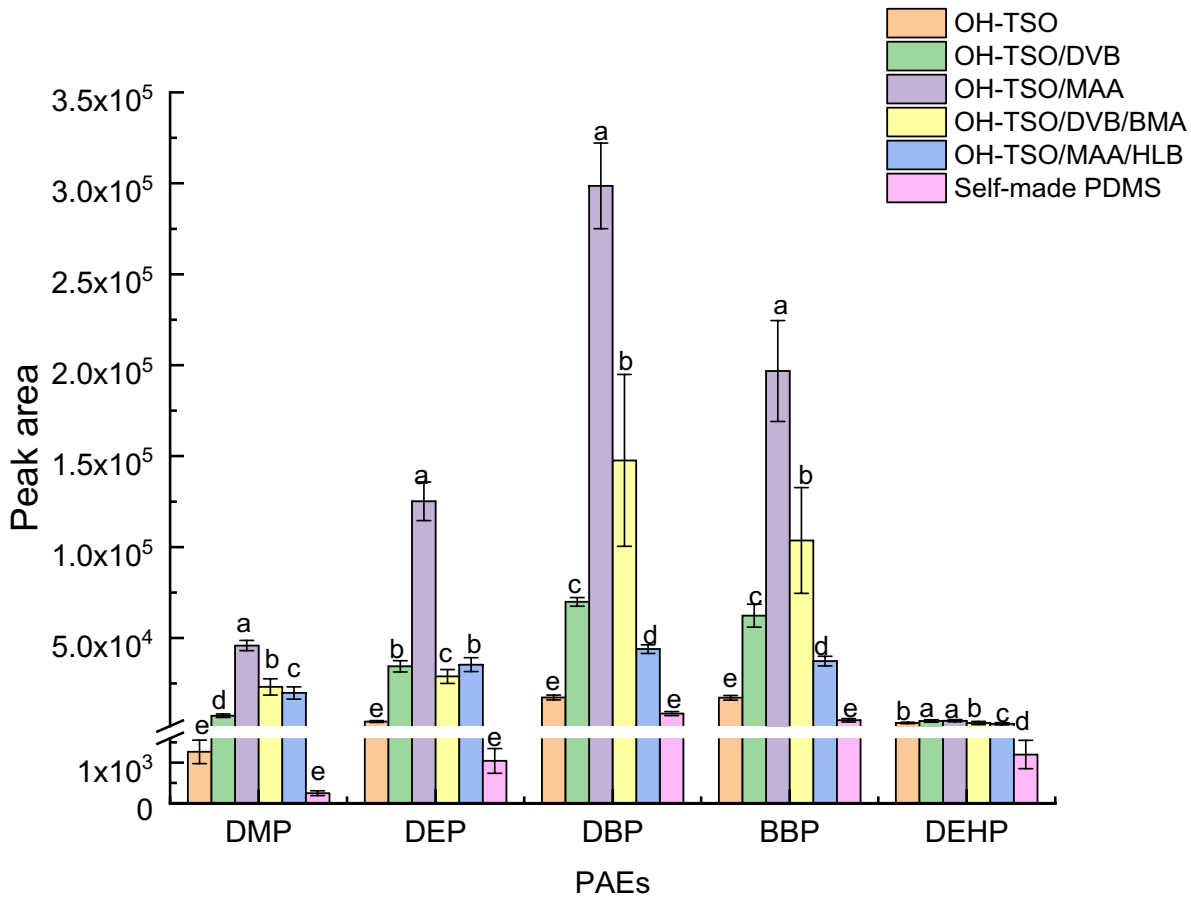


Table 2 The linear ranges, coefficients of determination $\left(R^{2}\right)$, precision (RSD), LOD, and LOQ

\begin{tabular}{lclllc}
\hline Analytes & Linear range $\left(\mu \mathrm{g} \mathrm{kg}^{-1}\right)$ & $R^{2}$ & $\mathrm{LOD}^{\mathrm{a}}\left(\mu \mathrm{g} \mathrm{kg}^{-1}\right)$ & $\mathrm{LOQ}^{\mathrm{b}}\left(\mu \mathrm{g} \mathrm{kg}^{-1}\right)$ & $\mathrm{RSD}^{\mathrm{c}}(\%, n=5)$ \\
\hline DMP & $1.25-62.5$ & 0.9989 & 0.655 & 1.250 & 10.29 \\
DEP & $0.5-25$ & 0.9990 & 0.233 & 0.500 & 9.06 \\
DBP & $0.25-12.5$ & 0.9994 & 0.025 & 0.084 & 8.74 \\
BBP & $0.375-18.5$ & 0.9969 & 0.170 & 0.375 & 14.20 \\
DEHP & $2.5-125$ & 0.9986 & 0.728 & 2.426 & 9.05 \\
\hline
\end{tabular}

${ }^{a}$ The LOD and ${ }^{b}$ LOQ were obtained based on the standard deviation $(\delta)$ of the responses in six replicated blank extractions and the slope $(\mathrm{S})$ of the calibration curve according to the equations $\mathrm{LOD}=3 \delta / \mathrm{S}$ and $\mathrm{LOQ}=10 \delta / \mathrm{S}$

${ }^{\mathrm{c}}$ Spiking level: $6.25 \mu \mathrm{g} \mathrm{kg}^{-1}$ for DMP, $2.5 \mu \mathrm{g} \mathrm{kg}^{-1}$ for DEP, $1.25 \mu \mathrm{g} \mathrm{kg}^{-1}$ for DBP, $1.875 \mu \mathrm{g} \mathrm{kg}^{-1}$ for BBP, and $12.5 \mu \mathrm{g} \mathrm{kg}^{-1}$ for DEHP
As for the sol-gel OH-TSO/DVB/BMA fiber, the coating was structurally loose and easy to fall off, and as a result, the reusability was not good. The service life of the homemade PDMS coating was 27 times, but the repeatability was poor, and the RSDs of 4 PAEs were all higher than $20 \%$.

The applications of sol-gel materials in biomedical field [35] suggest their excellent matrix compatibility in blood, which means that the materials are less likely to hurt themselves as well as the blood components. At the same time, sol-gel technology gives the coating a three-dimensional network structure and nano-scale pores, which can isolate biological macromolecules from the surface. DVB, BMA, and other ingredients are usually added to improve the extraction performance of the coating, but it seems that they do not contribute much to the service life of the coating, which may be related to many factors. On the one hand,

Table 3 Recoveries and precision (RSD) of PAEs in spiked whole blood samples

\begin{tabular}{llrc}
\hline Analytes & $\begin{array}{l}\text { Spiking levels } \\
\left(\mu \mathrm{kg}^{-1}\right)\end{array}$ & Recovery $(\%)^{\mathrm{a}}$ & $\mathrm{RSD}(\%, n=3)$ \\
\hline DMP & 2.5 & 96.28 & 6.58 \\
& 12.5 & 97.73 & 9.93 \\
& 62.5 & 100.92 & 6.18 \\
DEP & 1 & 104.19 & 10.99 \\
& 5 & 108.67 & 15.85 \\
& 25 & 99.85 & 9.97 \\
DBP & 0.5 & 119.89 & 7.18 \\
& 2.5 & 93.91 & 2.48 \\
& 12.5 & 99.73 & 3.52 \\
BBP & 0.75 & 82.93 & 6.46 \\
& 3.75 & 108.71 & 15.08 \\
& 18.75 & 101.02 & 5.08 \\
DEHP & 5 & 115.96 & 11.84 \\
& 25 & 86.01 & 13.43 \\
& 125 & 100.09 & 10.87 \\
\hline
\end{tabular}

${ }^{\mathrm{a}} \mathrm{A} t$-test was applied for statistical processing; the results indicate that there is no difference between the spiked concentration and the measurement result $(p>0.05)$
PAEs require long extraction time and high desorption temperature; on the other hand, these components are small molecules, their polymerization conditions are unclear, and their thermal stability is also poorer than that of PDMS, OH-TSO, and other silylating agents. Generally speaking, the sol-gel coated fibers showed acceptable matrix compatibility in undiluted plasma.

Figure 2 compares the extraction capacity of different coatings. As expected, the extraction capacity of sol-gel OH-TSO coating was higher than that of the self-made PDMS due to the outstanding properties of sol-gel technology. MAA, DVB, BMA, and HLB all could enhance the extraction capacity to PAEs. OT-TSO/MAA fiber achieved the highest extraction capacity owing to the strong polarity of MAA. The HLB coating was unexpectedly poor and later

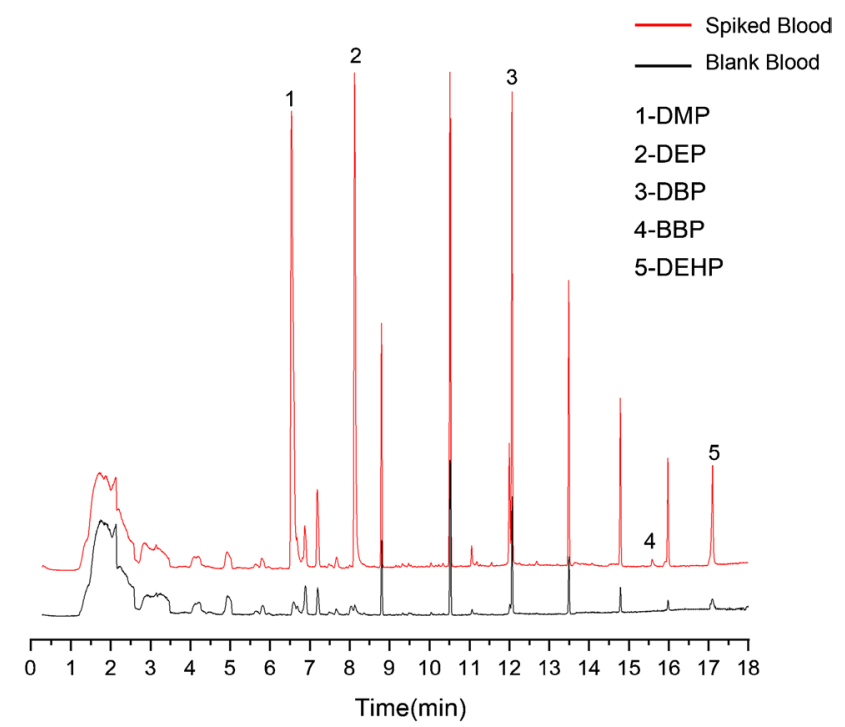

Fig. 3 Chromatograms of 5 PAEs in spiked and unspiked whole blood by DI-SPME/GC-MS. Spiked concentration: DMP, $62.5 \mu \mathrm{g} \mathrm{kg}^{-1}$; DEP, $25 \mu \mathrm{g} \mathrm{kg}^{-1}$; DBP, $12.5 \mu \mathrm{g} \mathrm{kg}^{-1}$; BBP, $18.75 \mu \mathrm{g} \mathrm{kg}^{-1}$; DEHP, $125 \mu \mathrm{g} \mathrm{kg}^{-1}$. Extraction and desorption conditions: extraction time, $30 \mathrm{~min}$; extraction temperature, $37{ }^{\circ} \mathrm{C}$; desorption temperature, $280{ }^{\circ} \mathrm{C}$; desorption time, $10 \mathrm{~min}$; cleaning condition, method 5 
Table 4 Comparison of the proposed DI-SPME/GC-FID method with other methods reported in literatures for determination of PAEs in blood

\begin{tabular}{|c|c|c|c|c|c|c|}
\hline Methods & Sample pretreatment & Linear range & LOD & $R^{2}$ & RSD (\%) & Ref \\
\hline $\begin{array}{l}\text { Dispersive magnetic } \\
\text { SPE-GC/MS }\end{array}$ & $\begin{array}{l}\text { Plasma was mixed with } \mathrm{HCl}(37 \% \\
\text { w/w) and TFA to precipitate } \\
\text { proteins. Afterwards, the resultant } \\
\text { solution was vortexed and then } \\
\text { centrifuged. The supernatant was } \\
\text { separated and diluted with ultrapure } \\
\text { water }\end{array}$ & $0.5-200 \mathrm{~g} \mathrm{~L}^{-1}$ & $0.08-0.15 \mathrm{~g} \mathrm{~L}^{-1}$ & $0.9977-0.9993$ & $2.50-9.50$ & [46] \\
\hline SPE-GC/MS & $\begin{array}{l}\text { Serum was loaded onto and passed } \\
\text { through the cartridge. After wash- } \\
\text { ing with } 5 \% \text { acetonitrile aqueous } \\
\text { solution, the analytes were eluted } \\
\text { with acetonitrile and collected in } \\
\text { clean glass tubes. The eluent was } \\
\text { evaporated to dryness under a } \\
\text { gentle stream of nitrogen at } 45^{\circ} \mathrm{C} \text {. } \\
\text { The residue was reconstituted for } \\
\text { analysis in acetonitrile }\end{array}$ & $5-1000 \mathrm{ng} \mathrm{mL}^{-1}$ & $2.3-13 \mathrm{ng} \mathrm{mL}^{-1}$ & $0.9869-0.9973$ & $5.2-13.4$ & [47] \\
\hline $\begin{array}{l}\text { Dispersive liquid- } \\
\text { liquid microextrac- } \\
\text { tion-GC/MS }\end{array}$ & $\begin{array}{l}0.2 \mathrm{mg} \text { trichloroacetic acid removes } \\
\text { proteins from } 10 \mathrm{~mL} \text { plasma and } \\
\text { yields } 7 \mathrm{~mL} \text { supernatant. Then the } \\
\text { supernatant was extracted with } \\
750 \mu \mathrm{L} \text { acetonitrile containing } 10 \\
\mu \mathrm{L} \text { chlorobenzene. This solution } \\
\text { ( } \mathrm{pH}=5 \text { ) was centrifuged for } 5 \mathrm{~min} \\
\text { at } 4500 \mathrm{rpm} \text {. After centrifuging, the } \\
\text { dispersed fine droplets of extracting } \\
\text { solvent sedimented at the bottom } \\
\text { of the test tube. The volume of the } \\
\text { sedimented phase was determined } \\
\text { by a } 100 \mu \mathrm{L} \text { microsyringe. } 2 \mu \mathrm{L} \text { of } \\
\text { the sedimented phase was removed } \\
\text { by a } 10 \mu \mathrm{L} \text { microsyringe and } \\
\text { injected into GC }\end{array}$ & $50-1000 \mathrm{ng} \mathrm{mL}^{-1}$ & $1.5-2.5 \mathrm{ng} \mathrm{mL}^{-1}$ & 0.996-0.998 & $3.2-4.1$ & [48] \\
\hline DI-SPME-GC/MS & $\begin{array}{l}\text { The serum lyophilized content of } \\
\text { Moni-Trol vials was reconstituted } \\
\text { with water and homogenized at } \\
\text { room temperature. After reconstitu- } \\
\text { tion, } 2 \text { mL samples were spiked } \\
\text { with phthalate ester standard } \\
\text { solution. After } 1 \text { h of contact time, } \\
\text { samples were pretreated by adding } \\
0.5 \text { mL of acetonitrile. Extraction } \\
\text { was performed by direct-immersion } \\
\text { SPME }\end{array}$ & $30-4000 \mathrm{ppb}$ & $15 \mathrm{pg} \mu \mathrm{L}^{-1}$ & $0.978-0.993$ & $<4$ & [49] \\
\hline DI-SPME-GC/FID & $\begin{array}{l}\text { Anticoagulated whole blood was } \\
\text { directly put to SPME }\end{array}$ & $0.25-125 \mu \mathrm{g} \mathrm{kg}^{-1}$ & $\begin{array}{l}0.025- \\
0.728 \mu \mathrm{g} \mathrm{kg}^{-1}\end{array}$ & $0.9968-0.9994$ & $\begin{array}{l}8.74- \\
14.20\end{array}$ & $\begin{array}{l}\text { Present } \\
\text { method }\end{array}$ \\
\hline
\end{tabular}

it was found that the particles were too large. After the particles were ground to a size of $1-5 \mu \mathrm{m}$, the coating had better extraction efficiency (the data were not shown in this paper).

\section{Lifetime of sol-gel OH-TSO/MAA coating in whole blood}

Due to its excellent performance to PAEs in plasma, $\mathrm{OH}-$ TSO/MAA fiber was tested in whole blood, and the results are given in Fig. S4 (Supplementary information). The
OH-TSO/MAA fiber could be continuously used for 22 times in whole blood with RSD between 7.27 and $14.84 \%$ for 5 PAEs. After cleaning, the coating surface was still smooth and clean under the microscope. Recently, a new SPME coating, namely fluoropolymer/hydrophilic-lipophilic balance, was developed and it could be used for 15 consecutive extractions in whole blood samples [23]. The comparison demonstrates the good matrix compatibility of OH-TSO/MAA fiber in whole blood, indicating its application prospect as probes 
Table 5 Comparison of the concentrations of $C$ obtained by the two sampling methods

\begin{tabular}{|c|c|c|c|c|}
\hline \multirow[t]{2}{*}{ Analytes } & \multirow{2}{*}{$\begin{array}{l}\text { Spiking levels } \\
\left(\mu \mathrm{kg}^{-1}\right)\end{array}$} & \multicolumn{2}{|l|}{$C\left(\mu \mathrm{g} \mathrm{kg}^{-1}\right)$} & \multirow[t]{2}{*}{$P^{\mathrm{a}}$} \\
\hline & & In vivo SPME & DI-SPME & \\
\hline \multirow[t]{4}{*}{ DMP } & 1.25 & $15.74 \pm 0.67$ & $1.51 \pm 0.35$ & $<0.05$ \\
\hline & 6.25 & $17.12 \pm 0.34$ & $7.81 \pm 1.90$ & $<0.05$ \\
\hline & 25 & $21.52 \pm 1.52$ & $20.45 \pm 2.56$ & $>0.05$ \\
\hline & 41.67 & $32.44 \pm 3.80$ & $31.83 \pm 5.02$ & $>0.05$ \\
\hline \multirow[t]{4}{*}{ DEP } & 0.5 & $1.69 \pm 0.47$ & $0.75 \pm 0.56$ & $>0.05$ \\
\hline & 2.5 & $2.53 \pm 0.19$ & $2.53 \pm 0.48$ & $>0.05$ \\
\hline & 10 & $6.92 \pm 0.82$ & $8.73 \pm 1.05$ & $>0.05$ \\
\hline & 16.67 & $14.35 \pm 0.95$ & $16.20 \pm 2.24$ & $>0.05$ \\
\hline \multirow[t]{4}{*}{ DBP } & 0.25 & $2.08 \pm 0.11$ & $0.31 \pm 0.14$ & $<0.05$ \\
\hline & 1.25 & $2.97 \pm 0.26$ & $1.64 \pm 0.44$ & $<0.05$ \\
\hline & 5 & $5.31 \pm 0.22$ & $4.74 \pm 0.49$ & $>0.05$ \\
\hline & 8.33 & $7.32 \pm 0.67$ & $8.10 \pm 0.71$ & $>0.05$ \\
\hline \multirow[t]{4}{*}{ BBP } & 0.375 & $3.55 \pm 0.36$ & $0.39 \pm 0.47$ & $<0.05$ \\
\hline & 1.875 & $3.41 \pm 0.13$ & $2.25 \pm 0.77$ & $>0.05$ \\
\hline & 7.5 & $5.69 \pm 0.58$ & $5.00 \pm 0.69$ & $>0.05$ \\
\hline & 12.17 & $8.25 \pm 0.70$ & $9.49 \pm 1.60$ & $>0.05$ \\
\hline \multirow[t]{4}{*}{ DEHP } & 2.5 & $47.83 \pm 1.49$ & $2.16 \pm 0.22$ & $<0.05$ \\
\hline & 12.5 & $51.45 \pm 3.11$ & $13.04 \pm 2.91$ & $<0.05$ \\
\hline & 50 & $57.98 \pm 1.84$ & $40.45 \pm 3.54$ & $<0.05$ \\
\hline & 83.33 & $77.44 \pm 3.84$ & $76.73 \pm 6.81$ & $>0.05$ \\
\hline
\end{tabular}

${ }^{\text {a } A ~} t$-test is applied for statistical processing; $p<0.05$ indicates that there is a significant difference in the groups, and $p>0.05$ indicates that there is no significant difference

for blood sampling. Nonetheless, measures should be taken to further improve the lifecycles of sol-gel SPME coatings.

\section{Establishment and evaluation of DI-SPME/GC-FID method for PAEs in whole blood using sol-gel OH-TSO/MAA fiber}

In order to further study the performance of sol-gel coatings in whole blood, a DI-SPME/GC-FID method was established to detect PAEs in blood using sol-gel $\mathrm{OH}-$ TSO/MAA fiber after several parameters associated with SPME efficiency were optimized (Fig. S5, Supplementary information).

As shown in Table 2, the linear ranges were achieved in $\mu \mathrm{g}$ $\mathrm{kg}^{-1}$ levels with good coefficients of determination for all analytes. The precision, expressed as RSD, gave satisfactory outcomes, ranging from 8.74 to $14.20 \%$. The LODs were between 0.025 and $0.728 \mu \mathrm{g} \mathrm{kg}^{-1}$. The method was used to analyze PAEs in unspiked pig blood sample. The result showed that the sample was positive and the concentrations were $0.46 \mu \mathrm{g} \mathrm{kg}^{-1}$ for DMP, $0.84 \mu \mathrm{g} \mathrm{kg}^{-1}$ for DEP, $0.41 \mu \mathrm{g} \mathrm{kg}^{-1}$ for DBP, and $1.61 \mu \mathrm{g} \mathrm{kg}^{-1}$ for DEHP. BBP was not detected. And then, recovery tests were performed in order to study accuracy. As shown in Table 3, the recoveries of five PAEs in the spiked blood were between 82.93 and $119.89 \%$ with RSD $\leq 15.85 \%$, revealing the good accuracy and repeatability of the method, and meet the FDA criteria of bioanalytical validation guidelines [45]. In order to confirm the result, both a spiked sample and an unspiked sample were analyzed by GC-MS. Figure 3 shows the chromatograms. In the blank sample, both of the two small peaks at the retention time of peak 1 are DMP, the small peak on the left of peak 2 is diethyltoluamide, and the interfering peak on the left of peak 3 is octadecamethylcyclononasiloxane (from the column coating). They are difficult to be separated from the analytes, and cause influence on the recovery, but have little effect when the concentrations of analytes are high.

Table 4 compares the sample pretreatments and analytical performance of the proposed DI-SPME/GC-FID method and other methods reported in literature for determination of PAEs in blood samples. The proposed method is rapid, simple, sensitive, and does not need expensive instruments or tedious procedures. The LODs were similar with or rather lower than those obtained by the reported methods.

\section{The potential of sol-gel coatings for in vivo SPME/ GC}

SPME is a non-exhaustive extraction technique in which only a small part of analytes is removed from the sample matrix. Therefore, the results of SPME need to be calibrated so as to get the accurate concentrations. The existing calibration methods of SPME have been summarized and discussed in other document [1]. Thereinto, the sampling rate calibration approach is widely used for in vivo sampling [1, 7]. It assumes that within a linear model of an extraction process, the rate of mass transfer (or sampling rate) remains constant throughout the duration of sampling, and the relationship between the concentration of target analyte in the sample matrix and the extracted amount of analyte can be expressed by Eq. (1). The sampling rates for targets can be pre-determined under laboratory conditions, and then can be directly used for quantification during in vivo analysis.

Figs. S6 and S7 (Supplementary information) investigate the sampling conditions, and the $R_{s}$ were obtained and presented in Table S3 (Supplementary information).

Four levels of PAEs standard solutions were spiked to whole blood samples. After overnight incubation at $4{ }^{\circ} \mathrm{C}$, the samples were analyzed by DI-SPME/GC method and in vivo SPME/GC method, respectively. As shown in Table 5, for the two high spiking levels, the detected values by in vivo sampling were close to the spiked ones, but for the low spiking levels, the method was noneffective. However, the DI-SPME sampling was always effective for all of the concentrations. According to the current research, $R_{s}$ may be related to the 
concentrations of analytes in the sample. The concentration of $R_{S}$ calculated (Table $\mathrm{S} 3$, Supplementary information) was between the two high concentrations in Table 5, and the concentrations obtained by these rates were more accurate. Follow-up studies will be carried out for more accurate quantification for in vivo sampling.

\section{Conclusion}

It's the first time to systematically and comprehensively assess the matrix compatibility of sol-gel coatings in blood samples. The model analytes are PAEs. The extraction time is long and the desorption temperature is high. These factors are not conducive to service life evaluation. Even so, the lifetime of these fibers was similar or longer than other fibers reported in literature, indicating their good matrix compatibility in blood. The successful application of these coatings in whole blood and artificial veins demonstrates the great potential of sol-gel SPME coatings in in vivo sampling, which is a very important technique for animal research. In the long run, it is necessary to further extend the service life of the coatings, and at the same time, it is necessary to develop and improve more effective quantitative correction methods for in vivo sampling.

Supplementary Information The online version contains supplementary material available at https://doi.org/10.1007/s00216-022-03890-2.

Acknowledgements The authors thank Prof. Gang Fan and his graduate students for fruitful discussion about the experiment of GC-MS.

Author contribution Xiao-Wei Zhang, experimental work, data processing, and manuscript writing; Yao-Juan Chu, experimental work; Yu-Hao Li, experimental work and manuscript writing; Xiu-Juan Li, strategy development and manuscript writing.

Funding This work was kindly supported by Hubei Agricultural Sciences and Technology Innovation Center (2019-620-000-001-31) and the Fundamental Research Funds for the Central Universities (2662020SPPY015).

\section{Declarations}

Ethics approval All experimental procedures were approved by the Animal Ethics Committee of the Huazhong Agricultural University.

\section{Conflict of interest The authors declare no competing interests.}

Animal welfare All applicable international, national, and/or institutional guidelines for the care and use of animals were followed. The blood samples used in this work were obtained from a slaughter house that provides pork for supermarkets. As this blood was not collected specifically for this study, this work was exempted from IACUC approval.

\section{References}

1. Ouyang GF, Vuckovic D, Pawliszyn J. Nondestructive sampling of living systems using in vivo solid-phase microextraction. Chem Rev. 2011;111(4):2784-814

2. Cudjoe E, Bojko B, Togunde P, Pawliszyn J. In vivo solidphase microextraction for tissue bioanalysis. Bioanalysis. 2012;4(21):2605-19.

3. Xu JQ, Chen GS, Huang SY, Qiu JL, Jiang RF, Zhu F, et al. Application of in vivo solid-phase microextraction in environmental analysis. TrAC, Trends Anal Chem. 2016;85:26-35.

4. Kou XX, Chen GS, Huang SM, Ye YX, Ouyang GF, Gan J, et al. In vivo sampling: a promising technique for detecting and profiling endogenous substances in living systems. J Agric Food Chem. 2019;67(8):2120-6.

5. Riboni N, Fornari F, Bianchi F, Careri M. Recent advances in in vivo SPME sampling. Separations. 2020;7(1):6.

6. Souza-Silva ÉA, Reyes-Garces N, Gomez-Rios GA, Boyaci E, Bojko B, Pawliszyn J. A critical review of the state of the art of solid-phase microextraction of complex matrices III. Bioanalytical and clinical applications. TrAC, Trends Anal Chem. 2015;71:249-64.

7. Zhang X-F, Zhao L-L, Huang M-Q, Li X-J, Pan S-Y. In situ real-time tracing of organophosphorus pesticides in apples by solid-phase microextraction with developed sampling-rate calibration. Molecules. 2019;24(24):4444.

8. Lord HL, Zhang X, Musteata FM, Vuckovic D, Pawliszyn J. In vivo solid-phase microextraction for monitoring intravenous concentrations of drugs and metabolites. Nat Protoc. 2011;6(6):896-924.

9. Mirnaghi FS, Pawliszyn J. Reusable solid-phase microextraction coating for direct immersion whole-blood analysis and extracted blood spot sampling coupled with liquid chromatography-tandem mass spectrometry and direct analysis in real-time tandem mass spectrometry. Anal Chem. 2012;84(19):8301-9.

10. Baumer A, Escher BI, Landmann J, Ulrich N. Direct sample introduction GC-MS/MS for quantification of organic chemicals in mammalian tissues and blood extracted with polymers without clean-up. Anal Bioanal Chem. 2020;412(26):7295-305.

11. Vuckovic D, Cudjoe E, Hein D, Pawliszyn J. Automation of solid-phase microextraction in high-throughput format and applications to drug analysis. Anal Chem. 2008;80(18):6870-80.

12. Lord HL, Grant RP, Walles M, Incledon B, Fahie B, Pawliszyn JB. Development and evaluation of a solid-phase microextraction probe for in vivo pharmacokinetic studies. Anal Chem. 2003;75(19):5103-15.

13. Musteata FM, Musteata ML, Pawliszyn J. Fast in vivo microextraction: a new tool for clinical analysis. Clin Chem. 2006;52(4):708-15.

14. Musteata FM, Lannoy I, Gien B, Pawliszyn J. Blood sampling without blood draws for in vivo pharmacokinetic studies in rats. J Pharm Biomed Anal. 2008;47(4-5):907-12.

15. Szultka M, Krzeminski R, Jackowski M, Buszewski B. Simultaneous determination of selected chemotherapeutics in human whole blood by molecularly imprinted polymers coated solid phase microextraction fibers and liquid chromatography-tandem mass spectrometry. J Chromatogr B. 2013;940:66-76.

16. Zhang X, Es-haghi A, Musteata FM, Ouyang G-F, Pawliszyn J. Quantitative in vivo microsampling for pharmacokinetic studies based on an integrated solid-phase microextraction system. Anal Chem. 2007;79(12):4507-13.

17. Es-haghi A, Zhang X, Musteata FM, Bagheri H, Pawliszyn J. Evaluation of bio-compatible poly(ethylene glycol)-based solidphase microextraction fiber for in vivo pharmacokinetic studies of diazepam in dogs. Analyst. 2007;132(7):672-8 
18. Reyes-Garces N, Bojko B, Hein D, Pawliszyn J. Solid phase microextraction devices prepared on plastic support as potential single-use samplers for bioanalytical applications. Anal Chem. 2015;87(19):9722-30.

19. Bessonneau V, Zhan Y-W, De Lannoy IAM, Saldivia V, Pawliszyn J. In vivo solid-phase microextraction liquid chromatography-tandem mass spectrometry for monitoring blood eicosanoids time profile after lipopolysaccharide-induced inflammation in SpragueDawley rats. J Chromatogr A. 2015;1424:134-8.

20. Rickert DA, Gómez-Ríos GA, Nazdrajic E, Tascon M, Kulasingam V, Pawliszyn J. Evaluation of a coated blade spray-tandem mass spectrometry assay as a new tool for the determination of immunosuppressive drugs in whole blood. Anal Bioanal Chem. 2020;412:5067-76.

21. Reyes-Garces N, Alam MN, Pawliszyn J. The effect of hematocrit on solid-phase microextraction. Anal Chim Acta. 2018;1001:40-50.

22. Reyes-Garces N, Bojko B, Buszewski B. High throughput quantification of prohibited substances in plasma using thin film solid phase microextraction. J Chromatogr A. 2014;1374:40-9.

23. Gionfriddo E, Boyaci E, Pawliszyn J. New generation of solidphase microextraction coatings for complementary separation approaches: a step toward comprehensive metabolomics and multiresidue analyses in complex matrices. Anal Chem. 2017;89(7):4046-54.

24. Hou YJ, Deng JW, He KL, Chen C, Yang YY. Covalent organic frameworks-based solid-phase microextraction probe for rapid and ultrasensitive analysis of trace per- and polyfluoroalkyl substances using mass spectrometry. Anal Chem. 2020;92(15):10213-7.

25. Wu SJ, Cai CC, Cheng J, Cheng M, Zhou HB, Deng JL. Polydopamine/dialdehyde starch/chitosan composite coating for in-tube solid-phase microextraction and in-situ derivation to analysis of two liver cancer biomarkers in human blood. Anal Chim Acta. 2016;935:113-20.

26. Wu Q, Wu DQ, Guan YF. In vivo fast equilibrium microextraction by stable and biocompatible nanofiber membrane sandwiched in microfluidic device. Anal Chem. 2013;85(23):1524-31.

27. Zilfidou E, Kabir A, Furton KG, Samanidou V. An improved fabric phase sorptive extraction method for the determination of five selected antidepressant drug residues in human blood serum prior to high performance liquid chromatography with diode array detection. J Chromatogr B. 2019;1125:121720.

28. Mu L, Hu XG, Wen JP, Zhou QX. Robust aptamer sol-gel solid phase microextraction of very polar adenosine from human plasma. J Chromatogr A. 2013;1279:7-12.

29 El-Beqqali A, Andersson LI, Jeppsson AD, Abdel-Rehim M. Molecularly imprinted polymer-sol-gel tablet toward micro-solid phase extraction: II. Determination of amphetamine in human urine samples by liquid chromatography-tandem mass spectrometry. J Chromatogr B. 2017;1063:130-5.

30. Moein MM, Jabbar D, Colmsjö A, Abdel-Rehim M. A needle extraction utilizing a molecularly imprinted-sol-gel xerogel for online microextraction of the lung cancer biomarker bilirubin from plasma and urine samples. J Chromatogr A. 2014;1366:15-23.

31. Mirzajani R, Kardani F, Ramezani Z. Preparation and characterization of magnetic metal-organic framework nanocomposite as solid-phase microextraction fibers coupled with high-performance liquid chromatography for determination of non-steroidal antiinflammatory drugs in biological fluids and tablet formulation samples. Microchem J. 2019;144:270-84.

32. Piri-Moghadam H, Alam MN, Pawliszyn J. Review of geometries and coating materials in solid phase microextraction: opportunities, limitations, and future perspectives. Anal Chim Acta. 2017;984:42-65.

33. Amiri A. Solid-phase microextraction-based sol-gel technique. TrAC, Trends Anal Chem. 2016;75:57-74.
34. Wang Y-L, Gao Y-L, Wang P-P, Shang H, Pan S-Y, Li X-J. Sol-gel molecularly imprinted polymer for selective solid phase microextraction of organophosphorous pesticides. Talanta. 2013;115:920-7.

35. Owens GJ, Singh RK, Foroutan F, Alqaysi M, Han C-M, Mahapatra $\mathrm{C}$, et al. Sol-gel based materials for biomedical applications. Prog Mater Sci. 2016;77:1-79.

36. Dominguez-Romero E, Scheringer M. A review of phthalate pharmacokinetics in human and rat: what factors drive phthalate distribution and partitioning? Drug Metab Rev. 2019;51(3):314-29.

37. Vasiljevic T, Singh V, Pawliszyn J. Miniaturized SPME tips directly coupled to mass spectrometry for targeted determination and untargeted profiling of small samples. Talanta. 2019;199:689-97.

38. Seryapina AA, Shevelev OB, Moshkin MP, Markel AL. Parameters of blood flow in great arteries in hypertensive ISIAH rats with stress-dependent arterial hypertension. Bull Exp Biol Med. 2016;161(4):468-71.

39. Grandy JJ, Lashgari M, Vander Heide H, Poole J, Pawliszyn J. Introducing a mechanically robust SPME sampler for the on-site sampling and extraction of a wide range of untargeted pollutants in environmental waters. Environ Pollut. 2019;252:825-34.

40. Liu MM. Preparation of novel sol-gel coatings for solid-phase microextraction and its application. Ph. D Dissertation, Wuhan University, 2006.

41. Gao YL. Preparation and evaluation of molecularly imprinted polymers for solid-phase microextraction and their application to the analysis of pesticide residues. Master Dissertation, Huazhong Agricultural University, 2013.

42. Xiang XZ. Preparation of high-efficiency solid-phase microextraction coating for environmental organic pollutants and its application in in situ sampling of Zizania Latifolia. Master Dissertation, Huazhong Agricultural University, 2021.

43. Gionfriddo E, Gruszecka D, Li X-J, Pawliszyn J. Direct-immersion SPME in soy milk for pesticide analysis at trace levels by means of a matrix-compatible coating. Talanta. 2020;211:120746.

44. De Grazia S, Gionfriddo E, Pawliszyn J. A new and efficient solid phase microextraction approach for analysis of high fat content food samples using a matrix-compatible coating. Talanta. 2017;167:754-60.

45. Guidance for Industry, Bioanalytical Method Validation. US Department of Health and Human Services, Food and Drug Administration. 2018. http://www.fda.gov/downloads/Drugs/ GuidanceComplianceRegulatoryInformation/Guidances/UCM07 0107.pdf. Accessed May 2018.

46. Dargahi R, Ebrahimzadeh H, Asgharinezhad AA, Hashemzadeh A, Amini MM. Dispersive magnetic solid-phase extraction of phthalate esters from water samples and human plasma based on a nanosorbent composed of MIL-101(Cr) metal-organic framework and magnetite nanoparticles before their determination by GC-MS. J Sep Sci. 2018;41(4):948-57.

47. Guo Z-Y, Gai P-P, Duan J, Zhai J-X, Zhao S-S, Wang S, et al. Simultaneous determination of phthalates and adipates in human serum using gas chromatography-mass spectrometry with solidphase extraction. Biomed Chromatogr. 2010;24(10):1094-9.

48. Ebrahim K, Poursafa P, Amin MM. Development of a simple and valid method for the trace determination of phthalate esters in human plasma using dispersive liquid-liquid microextraction coupled with gas chromatography-mass spectrometry. J Sep Sci. 2017;40(22):4403-10.

49. Colon I, Dimandja JMD. High-throughput analysis of phthalate esters in human serum by direct immersion SPME followed by isotope dilution-fast GC/MS. Anal Bioanal Chem. 2004;380(2):275-83.

Publisher's note Springer Nature remains neutral with regard to jurisdictional claims in published maps and institutional affiliations. 